\title{
Promoção da autodeterminação em alunos com necessidades educativas especiais: treino vocacional na comunidade
}

\author{
Alda Melo*, Paula C. Neves**, Maria João Antunes*** \\ *Agrupamento de Escolas de Albergaria -a -Velha, ${ }^{* *}$ Escola Superior de Educação de Coimbra, ***Agrupamento de Escolas de \\ Coimbra Centro
}

\begin{abstract}
Resumo
O presente estudo de investigação-ação pretendeu implementar um programa de atividades na comunidade com três alunos de catorze anos, com Necessidades Educativas Especiais, que beneficiam do decreto-lei 3/2008, artigo $21^{\circ}$ - Currículo Específico Individual e frequentavam o oitavo ano de escolaridade. Pretendeu ser um estudo piloto partindo da seguinte premissa: a autodeterminação de alunos com NEE que beneficiam de Currículo Específico Individual poderá melhorar com a promoção de atividades de treino vocacional na comunidade. Para avaliar a autodeterminação dos sujeitos utilizou-se o "The Arc 'Self Determination Scale", of Wehmeyer, na sua versão portuguesa e adaptada à população-alvo. Os resultados obtidos indiciam que a participação dos alunos em atividades de treino vocacional na comunidade contribuiu para promover uma melhoria significativa do nível de autodeterminação estimulando a resiliência para a transição para a Vida pós-escolar.

Palavras-chave: Educação Especial, Autodeterminação, Alunos com Currículo Específico Individual, Transição para a vida pós-escolar.
\end{abstract}

\begin{abstract}
This investigation action-research study aimed implementing a programme with activities in the community with three students with fourteen years old. These students with Special Needs are included in the decreto-lei 3/2008, art. $21^{\circ}$-Specific Individual Curriculum and attend the 8 th grade. It aims to be a pilot study that begins with the goal: the self-determination with Special Needs Specific Individual Curriculum will improve with promoting vocational training with activities in the community? The intervention use's "The Arc 'Self Determination Scale", of Wehmeyer, adapted to the target population. The results indict that the students participation in the vocational training activities in the community contributed to promote a significant improvement to the level of self-determination stimulating the resilience transition to Life after school.

KeyWords: Special Education, Self-determination, Students with Specific Individual Curriculum, Transition plan to active life.
\end{abstract}

\section{Introdução}

A autodeterminação das pessoas com incapacidade é, cada vez mais, assumida como um direito e referida como um dos princípios fundamentais descritos na convenção sobre os Direitos das Pessoas com Deficiência, das Nações Unidas (Nações Unidas, 2007).
Promover a autodeterminação de alguém é contribuir para a melhoria da sua qualidade de vida, uma vez que estimula a sua capacidade e a oportunidade de fazer escolhas, tomar decisões, definir metas e objetivos pessoais. No caso da população com deficiência intelectual (DI), jovens com maiores competências de autodeterminação têm maiores possibilidades de obter sucesso na transição para a vida adulta, onde se inclui o emprego e a vida social (Whemeyer \& Abery, 2013), pelo que a promoção da autodeterminação de alunos com Currículo Específico Individual, reveste-se da maior importância uma vez que facilita a sua plena inclusão na escola e na sociedade. A inexistência de investigações a nível nacional sobre esta temática justifica a pertinência deste projeto de intervenção, que pretendeu evidenciar a importância do treino vocacional na comunidade enquanto elemento promotor da autodeterminação de alunos com Necessidades Educativas Especiais (NEE) que beneficiam de Currículo Específico Individual (CEI).

O conceito de Autodeterminação refere-se ao conjunto de comportamentos e habilidades que dotam as pessoas da capacidade de serem os principais agentes causais da sua própria vida, fazendo escolhas e tomando decisões, tendo em conta a sua qualidade de vida, livre de interferência ou influência externa (Wehmeyer \& Abery, 2013). Ser autodeterminado é assumir as próprias ações no mais alto nível de reflexão. Quando autodeterminada, a pessoa experimenta um senso de liberdade para fazer o que é interessante, pessoalmente importante e revigorante (Deci \& Ryan, 2000).

A crescente ênfase no conceito de autodeterminação do movimento de autodefesa dos indivíduos com incapacidade, associada à crença de que os indivíduos com DI não possuem as competências que se estendem para além da tomada de decisões simples (Wehmeyer \& Abery, 2013) contribuiu para o desenvolvimento de modelos teóricos empiricamente validados da autodeterminação e para a criação de instrumentos com base teórica sustentada, para a avaliação e promoção de comportamentos autodeterminados das pessoas com DI ao longo das suas vidas (Verdugo, Vicente, Fernández-Pulido, Gomez-Vela, Wehmeyer, \& Guillén, 2015). 
Um destes instrumentos é a Arc's Self-Determination Scale de Wehmeyer (1995) que será utlizada neste projeto.

\section{O conceito de autodeterminação}

A investigação no âmbito da autodeterminação é relativamente recente. Deci e Ryan, apresentaram a primeira teoria sobre autodeterminação em 1985, na qual defendem que todos os sujeitos apresentam uma "tendência inata para elaborar um sentido do eu (self) unificado, ainda que este seja também determinado por fatores ambientais" (1985, p.8). Estes autores partem da conjetura de que todos os comportamentos humanos são conduzidos por três necessidades psicológicas básicas, que constituem a base da automotivação do indivíduo e da integração da sua personalidade: a necessidade de autonomia, a necessidade de competência e a necessidade de relacionamento. Estas três necessidades, são aspetos fundamentais, uma vez que estão na base do desenvolvimento social e bem-estar pessoal (Deci \& Ryan, 2000).

Para Wehmeyer (1999) a autodeterminação é um constructo que não pode ser definido nem como um conjunto de comportamentos, nem como um traço de personalidade, mas deve ser equacionado enquanto característica da ação ou comportamento. A autodeterminação refere-se ao agir intencionalmente enquanto agente causal do seu futuro, fazendo escolhas, decidindo sobre aspetos relativos à sua qualidade de vida, livres de influência ou interferência externa, e integra quatro características: autonomia, empowerment psicológico, autorrealização e autorregulação.

A autonomia diz respeito à capacidade de cada um, num contexto de satisfação das suas necessidades, estabelecer objetivos e resolver os problemas que vão surgindo.

$\mathrm{O}$ empowerment psicológico refere-se à perceção de controlo, ou seja, às crenças nas suas capacidades, às expetativas de sucesso no domínio cognitivo, da personalidade e motivacional.

A autorrealização é a capacidade de organizar um caminho de vida com significado, baseado em objetivos pessoais.

A autorregulação refere-se à capacidade de autocontrolo, à capacidade de resolução de problemas e de estabelecimento de objetivos, bem como de estratégias para uma aprendizagem contínua.

Assim, um comportamento para ser considerado autodeterminado, deve refletir estas quatro características: ser autónomo, autorregulado, ser expressão de empowerment psicológico e resultar em autorrealização (Wehmeyer, 1999).

Ser autodeterminado envolve a expressão das necessidades próprias, a adaptação de objetivos e estratégias, bem como a criação de abordagens para resolução de problemas (Shogren, Palmer, Wehmeyer, Williams-Diehm, \& Little, 2012), fatores que para Deci e Ryan (1987) são determinantes para alcançar o bem-estar psicológico e promover a qualidade de vida.

Desenvolver a autodeterminação impulsiona a independência de cada cidadão, pelo que, a sua promoção em pessoas com DI, pode ser um dos grandes marcos do século XXI (Schalock \& Verdugo, 2010; Wehmeyer, Martin \& Sands, 2008). Os indivíduos com DI que conseguem melhor nível de autodeterminação obtêm melhores resultados durante o percurso escolar e transição para a vida ativa e adulta (Whemeyer \& Abery, 2013).

Desta forma, tornar acessível a aprendizagem da autodeterminação a todos os alunos, implica a participação conjunta de todos os agentes educativos, a família, a escola e a comunidade, ou seja, de todos os contextos que fazem parte da vida dos jovens (educativo, familiar, laboral, etc.) (Vived, Mullet, Orgaz, Sanz \& Amor, 2013)

A autodeterminação pode ser desenvolvida no contexto da diversidade de atividades escolares, fomentando a planificação e o desenvolvimento de competências que promovam os diferentes componentes da autodeterminação (autonomia, autorregulação, empowerment psicológico e autorrealização), bem como na criação de oportunidades de trabalho em contexto real.

\section{A qualidade de vida e a autodeterminação}

A qualidade de vida é um conceito multidimensional, com indicadores objetivos e subjetivos, universais e culturais, é influenciado por características pessoais e contextuais, e tem oito domínios centrais, a saber: desenvolvimento pessoal, autodeterminação, relações interpessoais, inclusão social, direitos, bem-estar emocional, bem-estar físico e bem-estar material (Schalock \& Verdugo, 2002). Para as pessoas com DI, a autodeterminação é considerada como o domínio mais importante da qualidade de vida (Simões, 2016).

Promover a autodeterminação influencia a melhoria da qualidade de vida. Na mesma linha é também possível identificar uma relação inversa, na medida em que a experiência de múltiplas facetas da vida (e.g. inclusão, relações interpessoais, direitos, oportunidades, participação ou bem-estar) aumenta as oportunidades de se efetuarem escolhas, tomarem decisões, definirem metas e objetivos pessoais, implicando, por sua vez, o desencadear de atitudes mais autodeterminadas no sujeito (Simões, 2016).

A aprendizagem e o desenvolvimento das capacidades implicadas na autodeterminação, revestem-se de especial importância pelo que devem iniciar-se cedo e prolongar-se ao longo da vida. Estas competências permitem que os alunos desenvolvam atitudes e crenças que lhes proporcionam uma participação ativa nas decisões, na resolução de problemas, nas propostas e no alcançar de objetivos, em suma, nas decisões referentes à sua própria qualidade de vida (Heller et al., 2011).

Neste contexto, a portaria 201-C publicada em julho de 2015 refere que existindo hoje uma grande preocupação com a qualidade de vida das pessoas com necessidades especiais, o processo de transição para a vida pós-escolar deve ter como preocupação essencial a preparação dos jovens para uma vida com qualidade. No entanto, o desenvolvimento da autodeterminação e, consequentemente a qualidade de vida, dependem em parte, das diversas oportunidades proporcionadas por cada um dos agentes e interlocutores ao longo da vida, família, escola, empresários e da sociedade em geral. 
No caso da escola, esta tem um papel relevante e deve apostar na transição para a vida pós-escolar como um processo contínuo, o que significa que, ao longo de todo o percurso escolar do aluno devem ser desenvolvidas atividades de promoção de autonomia e desenvolvimento de várias competências essenciais para a melhoria do nível de autodeterminação dos jovens com DI, e consequentemente para a melhoria da sua qualidade de vida. No caso da transição para a vida pós-escolar é importante criar oportunidades para que os jovens decidam por si próprios o caminho a seguir após a escolarização.

\section{Metodologia}

\section{Definição do problema}

A constatação dos baixos níveis de autodeterminação dos alunos com NEE e especificamente dos alunos com CEI motivaram o desenvolvimento deste projeto.

Questão de partida - Poderá a promoção de um treino vocacional com atividades na comunidade melhorar o nível de autodeterminação dos alunos com NEE que beneficiam de CEI?

\section{Objetivo}

O objetivo deste projeto foi promover a autodeterminação de alunos com NEE e com CEI, através de atividades de treino vocacional na comunidade.

\section{Instrumentos}

Os instrumentos utilizados na recolha de dados antes e depois da intervenção foram a Arc's Self-Determination Scale de Wehmeyer em (1995) e a entrevista semiestruturada.

Escala de Wehmeyer - A escala apresenta quatro secções: Autonomia, Autorregulação, Empowerment Psicológico e Autorrealização (Wehmeyer, 1995) (tabela1). Neste estudo utilizou-se (com permissão dos autores) a versão adaptada para a população portuguesa por Lança, Lebre, Freitas, Marques e Feijã (2006). De acordo com Wehmeyer e Metzler (1995) a autodeterminação é definida como uma construção que não poderá ser avaliada de forma direta.

Tabela 1

Domínios da escala de Autodeterminação

\begin{tabular}{lcc}
\hline Domínios & $\mathrm{N}^{\circ}$ Itens & Cotações \\
\hline Autonomia (A,B,C,D, E, F) & $1-32$ & 0 a 3 \\
Autorregulação & $33-41$ & 0 a 3 \\
Empowerment Psicológico & $42-57$ & $0 / 1$ \\
Autorrealização & $58-72$ & $0 / 1$ \\
\hline
\end{tabular}

O domínio da autonomia é avaliado em 32 itens divididos em quadro subdomínios: A -Independência: Rotina de cuidados pessoais e funções orientadas pela família (item 1 a 6); B - Independência: Interação com o meio(item 7 a 10); C - Agir em função das preferências, crenças, interesses e capacidades: atividades recreativas e de lazer (item 11 a 16); D - Agir em função das preferências, crenças, interesses e capacidades: envolvimento e interação na comunidade (item 17 a 21); E - Agir em função das preferências, crenças, interesses e capacidades: orientação vocacional (item 22 a 26); F Agir em função das preferências, crenças, interesses e capacidades: expressão pessoal (item 27 a 32). O domínio da autoregulação é avaliado por 9 itens, de 33 a 41; o domínio empowerment psicológico pelos itens 42 a 57 e a autorrealização pelos itens 58 a 72 . A autonomia e autoregulação são cotadas de 0 a 3 e o empowerment psicológico e a autorrealização entre 0 e 1 .

A determinação do nível de Autodeterminação dos alunos, foi calculada com base aos critérios definidos por Wehmeyer (1995): após o cálculo da pontuação bruta de todos os domínios e subdomínios, esta foi convertida em percentagem com recurso às tabelas de conversão fornecidas pelo questionário. $\mathrm{O}$ limiar da autodeterminação (positive percentage) é definido como igual ou superior a $70 \%$.

Entrevista - Foram realizadas entrevistas aos encarregados de educação (EE) dos participantes. O guião da entrevista, estruturado de acordo com os quatro domínios da escala de Wehmeyer, permitiu recolher informação de cada domínio dos três alunos.

\section{Participantes}

Participaram neste projeto três jovens do sexo masculino (aluno A, B, C) com NEE e que frequentavam a escola no oitavo ano de escolaridade. Todos beneficiavam do decreto-lei 3/2008, artigo $21^{\circ}$ Currículo Específico Individual e iriam iniciar o seu Plano Individual de Transição (PIT) no ano letivo seguinte.

\section{Procedimentos}

O projeto iniciou-se com a entrevista aos EE dos 3 alunos com o objetivo de identificar as suas perceções relativamente à autodeterminação dos seus educandos e as expetativas relativamente ao seu futuro profissional.

Num segundo momento foi realizada a avaliação dos níveis de autodeterminação dos participantes.

Realizaram-se diversas visitas a empresas na área geográfica circundante à escola com áreas de atividade diferentes e escolheram-se três - uma padaria-pastelaria, um restaurante e um minimercado, que viriam a ser os locais do treino vocacional.

Nos três meses seguintes cada aluno esteve dois dias por semana em cada uma das empresas, alternando entre os locais de três em três semanas. No final dos dois primeiros meses, foi-lhes pedido que escolhessem uma única empresa para lá ficarem no último mês. Durante o tempo que estiveram nas empresas os alunos realizaram tarefas específicas inerentes ao ramo de atividade de cada empresa, em parceria com os outros trabalhadores.

Após três meses de atividades laborais em empresas foi realizada uma segunda avaliação dos níveis de autodeterminação dos participantes e entrevistas aos EE.

Recolheram-se também informações junto das diretoras de turma e dos empresários que receberam os alunos. As informações recolhidas incidiram sobre 4 itens: realização do trabalho, comportamento social, 
assiduidade e pontualidade, autonomia e realização de tarefas.

\section{Apresentação e discussão dos resultados}

Os dados recolhidos na fase inicial do projeto evidenciaram, em todos os alunos, resultados baixos em cada um dos quatro domínios (autonomia, autodeterminação empowerment psicológico e autorrealização) (tabela 2) . Nenhum dos alunos atinge a percentagem considerada mínima $(70 \%)$ que caracteriza os sujeitos considerados autodeterminado (figura 1). Se considerarmos os resultados obtidos em cada domínio verificamos que o aluno A se distingue claramente dos outros, com um perfil de resultados muito mais baixos em todos os domínios. Os alunos B e C, apesar de terem resultados melhores que o aluno $\mathrm{A}$, registam também baixos níveis de autodeterminação.

Relativamente ao aluno $\mathrm{C}$, este tem resultados mais elevados no domínio do empowerment psicológico e no domínio da autorrealização, embora no global apresente um resultado (63\%) (tabela 2 ), que evidencia uma baixa autodeterminação.

Tabela 2

Cotação de cada aluno em cada domínio e no total de Autodeterminação na primeira avaliação

\begin{tabular}{llll}
\hline & Aluno A & Aluno B & Aluno C \\
\hline Autonomia & $11(11 \%)$ & $32(33 \%)$ & $32(33 \%)$ \\
Autoregulação & $3(14 \%)$ & $7(33 \%)$ & $6(29 \%)$ \\
$\begin{array}{l}\text { Empowerment } \\
\text { Psicológico }\end{array}$ & $5(31 \%)$ & $11(69 \%)$ & $10(63 \%)$ \\
Autorealização & $7(47 \%)$ & $13(87 \%)$ & $10(67 \%)$ \\
Autodeterminação & $\mathbf{2 6 ( 1 8 \% )}$ & $\mathbf{6 3 ( 4 3 \% )}$ & $\mathbf{5 8 ( 3 9 \% )}$ \\
\hline
\end{tabular}

Após a intervenção, constata-se na segunda avaliação que os resultados melhoraram em todos os domínios e em todos os alunos (tabela 3). Apenas o aluno A não atinge a percentagem considerada mínima (70\%), que caracteriza o sujeito considerado autodeterminado, embora tenha atingido um valor muito perto do esperado (65\%). Os outros dois alunos (A e B), obtiveram valores mais elevados de autodeterminação após a intervenção. O aluno B atingiu o nível de autodeterminação de (79\%) e o aluno C (77\%), pelo que, segundo a escala de Wehmeyer (1995) podem ser considerados autodeterminados.

Tabela 3

Cotação de cada aluno em cada domínio e no total de Autodeterminação na segunda avaliação

\begin{tabular}{lrrr}
\hline & Aluno A & Aluno B & Aluno C \\
\hline Autonomia & $55(57 \%)$ & $72(90 \%)$ & $72(75 \%)$ \\
Autoregulação & $15(71 \%)$ & $19(69 \%)$ & $14(67 \%)$ \\
$\begin{array}{l}\text { Empowerment } \\
\text { Psicológico }\end{array}$ & $16(100 \%)$ & $16(100 \%)$ & $16(100 \%)$ \\
Autorealização & $9(60 \%)$ & $13(87 \%)$ & $12(80 \%)$ \\
Autodeterminação & $\mathbf{9 6 ( 6 5 \% )}$ & $\mathbf{1 1 7 ( 7 9 \% )}$ & $\mathbf{1 1 4}(\mathbf{7 7 \%})$ \\
\hline
\end{tabular}

Relativamente ao nível de Autodeterminação o aluno A, é o aluno com a pontuação mais baixa dos três, quer na primeira, quer na segunda avaliação (figura 1). O aluno B obteve a pontuação mais elevada e os alunos B e C obtiveram uma pontuação muito próxima da totalidade da escala.

Em síntese, podemos verificar que todos os alunos melhoraram substancialmente os seus níveis de autodeterminação após o seu treino vocacional. Desta forma, considera-se que a intervenção foi muito benéfica para os três alunos, e que o seu objetivo principal foi atingido, ou seja, a intervenção melhorou significativamente o nível de autodeterminação destes alunos.

Houve uma evolução significativa em todos os domínios não se tendo verificado nenhum retrocesso em nenhum domínio (figura 1).

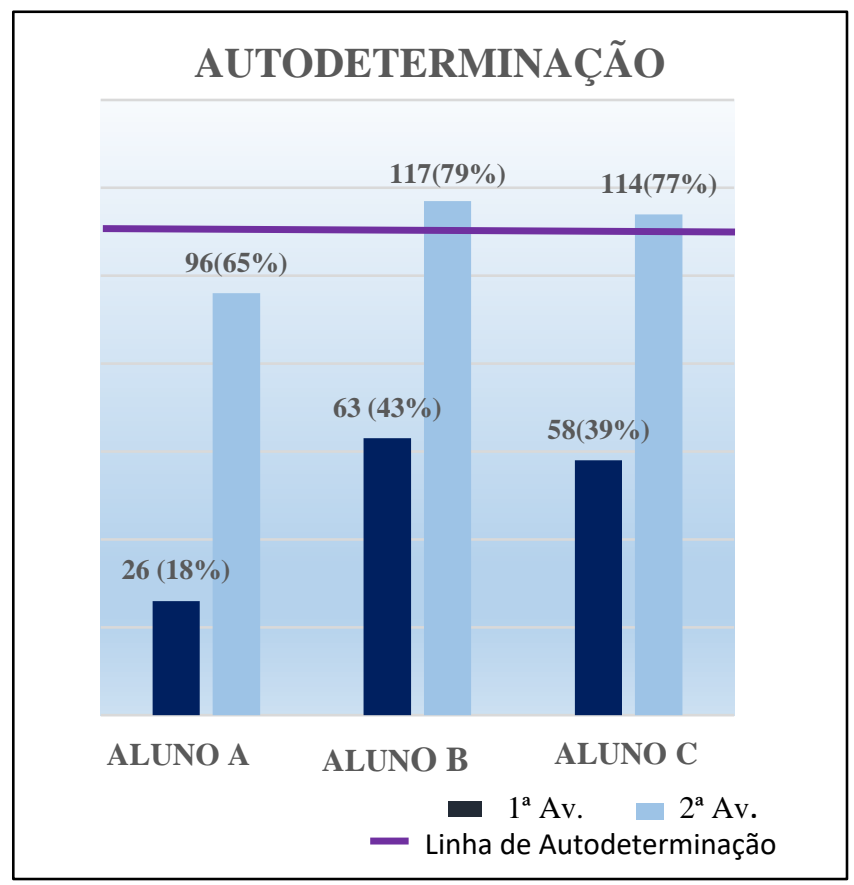

Figura 1- Nível de autodeterminação obtida pelos alunos nas duas avaliações

\section{Conclusões e implicações}

O objetivo principal deste projeto foi alcançado uma vez que a intervenção melhorou significativamente o nível de autodeterminação de todos os participantes em todos os domínios. Destaca-se que, os alunos B e C ficaram, apenas a $31(21 \%)$ e 34 pontos (23\%), respetivamente, do total da escala de 148 pontos (100\%), e o aluno A ficou a 56 pontos (38\%). Os alunos, que apresentavam um nível de autodeterminação muito baixo na primeira avaliação, aumentaram substancialmente na segunda avaliação.

Os resultados obtidos indiciam que a oportunidade de realizar um treino vocacional com atividades na comunidade possibilitou a estes alunos, com NEE e com CEI, melhorar o seu nível de autodeterminação proporcionando uma escolha importante para o seu futuro PIT. 
Este projeto foi um ponto de partida interessante para a realização de programas de treino vocacional na comunidade com alunos com CEI e, por sua vez, foi, ainda esclarecedor acerca da relevância da inclusão da autodeterminação na construção do CEI dos alunos com NEE, para que seja mais fácil proporcionar a transição para a vida pós escolar e a futura inserção no mercado de trabalho.

Assim, sendo a autodeterminação um elemento fulcral para a escolarização dos alunos com NEE (Arellano \& Peralta, 2013), a sua promoção deve ser equacionada desde cedo (Heller et al.,2011). Neste sentido, sugere-se a inclusão de competências relacionadas com a autodeterminação o mais cedo possível, principalmente na fase da adolescência, uma vez que nesta fase os alunos estão preparados para aprender competências de autorregulação e de estabelecimento de metas.

A aquisição de competências de autodeterminação permitirão ao aluno decidir sobre a sua forma de estar para conseguir a qualidade de vida e a participação social que deseja. Ao mesmo tempo, é também importante envolver e sensibilizar os EE para a importância da autodeterminação dos seus educandos, enquanto elemento facilitador da sua inclusão social.

Este projeto piloto permitiu a participação de cada um dos agentes e interlocutores importantes, nomeadamente a família, a escola, a comunidade. Porém, faltam ainda mais estudos que possam esclarecer quais os aspetos importantes no treino vocacional que influenciaram a melhoria da autodeterminação dos alunos.

No final deste projeto pretende-se planificar e organizar uma oficina de formação de professores de Educação Especial com o objetivo de partilhar os dados obtidos e proporcionar a elaboração de programas específicos de treino vocacional, que serão essenciais para a melhoria do nível de autodeterminação dos alunos.

\section{Referências}

Areallano R., \& Peralta F. (2013). Self-determination of young children with intellectual disability: understanding parents' perspectives. British Journal of Special Education 40 (4), 175-181. doi:10.1111/1467-8578.12037

Deci, E., \& Ryan, R. (1987). The support of autonomy and the control of behavior. Journal of Personality and Social Psychology, 53 (6), 1024-1037. doi:10.1037/0022-3514.53.6.1024

Deci, E., \& Ryan, R. (2000). Self-determination theory and the facilitation of intrinsic motivation, social development, and well-being. American Psychologist, 55 (1), 68-78. doi: 10.1037/0003-066X.55.1.68

Heller, T., Schindler, A., Palmer, S., Wehmeyer, M., Parent, W., Jenson, R., \& O' Hara, D. (2011). Self-determination across the life span: Issues and gaps. Exceptionality - A Special Education Journal, 19 (1), 31-45. doi: 10.1080/09362835.2011.537228

Lança, C., Lebre, F., Freitas, H., Marques, S., \& Feijão, S. (2006). Tradução e adaptação para população portuguesa da escala - Arc's Self-Determination Scale.(documento não impresso, cedido pelos autores)
Schalock, R., \& Verdugo, M. (2002). Quality of life for human service practitioners. Washington, DC: American Association on Mental Retardation.

Yeager, M. (2010). Intellectual disability: definition, classification, and systems of supports. (11th ed.). Washington, DC: American Association on Intellectual and Developmental Disabilities.

Shogren, K., Palmer, S., Wehmeyer, M., Williams-Diehm, K., \& Little, T. (2012). Effect of intervention with the self-determined learning model of instruction on access and goal attainment. Remedial and Special Education, 33 (5), 320-330. doi:10.1177/0741932511410072.

Simões, C. (2016). O direito à autodeterminação das pessoas com deficiência, Porto, APPC - FDUP.

Unesco (1994). Declaração de Salamanca e quadro de ação: conferência mundial para as necessidades educativas especiais: Acesso e Qualidade. Salamanca.

Verdugo, M., Vicente, E., Fernández-Pulido, R., Gomez-Vela, M., Wehmeyer, M., \& Guillén, V. (2015). A psychometric evaluation of the ARC-INICO Self-Determination Scale for adolescents with intellectual disabilities. International Journal of Clinical and Health Psychology, 15(2), 149-159. doi:10.1016/j.ijchp.2015.03.001.

Vived, E., Mullet, E., Orgaz, M., Sanz, A., \& Amor, A. (2013). Avanzando hacia la vida independiente: planteamientos educativos en jóvenes con discapacidad intelectual. Revista Española de Discapacidad,1(1), 119-138. doi:10.5569/2340-5104.01.01.06

Wehmeyer, M. (1995). The arc's self-determination scale: procedural guidelines: the arc of the United States and Michael Wehmeyer. Office of Special Education and Rehabilitative Services (ED), Washington, DC. Div. of Innovation and Development.

Wehmeyer, M. (1999). A functional model of self-determination: describing development and implementing instruction. Focus on Autism and Other Developmental Disabilities, 14 (1), 53-62. doi:10.1177/108835769901400107

Wehmeyer, M., Martin, J., \& Sands, D. (2008). Self-determination and students with developmental disabilities. H. P. Parette \& G. R. Peterson-Karlan (Eds.), Research-based practices in developmental disabilities (2nd ed.), 99-122. Austin, TX: ProEd doi:10.1177/1053815108324445

Wehmeyer, M., \& Abery, B. (2013). Self-determination and choice. Intellectual and Developmental Disabilities, 51(5), 399-411. doi: 10.1352/1934-9556-51.5.399

\section{Legislação e documentos normativos}

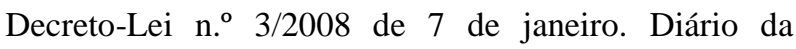
República n 4/2008-I Série. Ministério da Educação. Lisboa.

Portaria n. ${ }^{\text {o }}$ 275-A/2012 de 11 de setembro. Diário da República n. ${ }^{\circ}$ 176I/2012- I Série. Ministério da Educação.Lisboa.

Portaria 201-C/2015 de 10 de julho. Diário da República no 133/2015 -I Série. Ministério da Educação. Lisboa. 